\title{
Additional insights regarding aortic intramural hematoma
}

\author{
Kamal Kant Sahu, MD • Amos Lal, MD (1) - Ajay Kumar Mishra, MD
}

Received: 23 August 2019/Revised: 3 September 2019/Accepted: 3 September 2019/Published online: 1 October 2019

(c) Canadian Anesthesiologists' Society 2019

\section{To the Editor,}

We read with interest the recent article by Makhija et al. ${ }^{1}$ and would like to make a few comments to add perspective on aortic intramural hematoma (IMH). Makhija et al. outlined the usefulness of transesophageal echocardiography (TEE) in diagnosing an IMH where it becomes especially important when patients are too sick to be transported for a computed tomography (CT) scan. In contrast to CT, TEE is a readily available semi-invasive bedside procedure that can be easily performed in the operating room. It was not clear from the case description if the reported patient had had a CT scan.

IMH also needs to be differentiated from other acute aortic syndrome (AAS) subtypes. Penetrating aortic ulcers (PAUs) are often confused with ulcer like projections (ULPs). A PAU is basically a breach in the endothelium and internal elastic lamina, exposing the media (to varying degrees and depths). Depending on the depth of this penetration, a PAU can serve as a nidus for the development of an IMH, pseudo-aneurysm, or transmural rupture. While a PAU is believed to be the reason for the development of an IMH, the IMH can subsequently develop into an ULP. ${ }^{2,3}$ Furthermore, PAUs are strongly associated with aortic atherosclerosis, so are frequently

This letter is accompanied by a reply. Please see Can J Anesth 2020; 67: this issue.

K. K. Sahu, MD · A. K. Mishra, MD

Department of Medicine, Saint Vincent Hospital, Worcester, MA, USA

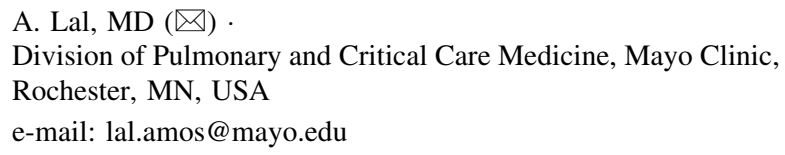

seen in the descending aorta. In contrast, ULPs have no such association, are frequently seen in ascending aorta, and are usually not present at initial CT. Any combination of more than one AAS (aortic dissection, IMH, ULP, and PAU) in an individual is considered a mixed-type aortic lesion and can be more challenging to interpret. For example, Makhija et al. described their case as having a PAU with an IMH. A few other mixed entities have been described, such as PAU with a secondary medial hematoma and an IMH with secondary ULPs. In a PAU with a secondary medial hematoma, there is ulceration of the atherosclerotic plaque through the intima and into the media with a variable amount of secondary bleed in the media adjacent to the intimal breech. Whereas in an IMH with secondary ULPs, during the process of hematoma expansion, stress severs the interlamellar connections from the media to the adventitia, which leads to formation of ULPs commonly visible at follow-up CT.

Kitai et al. reported a retrospective analysis of 170 patients with acute type B aortic dissection who were initially medically managed. They found that 62 out of 170 (36\%) patients developed ULP with maximum adverse aorta-related events associated with proximal descending ULPs within 30 days of disease onset. More importantly, they noted that ULP development was associated with worse survival rates compared with patients who did not develop ULP $(P=0.037){ }^{4}$

With regards to prognosis in PAUs, there is no clear risk stratification described, but a neck diameter $>10 \mathrm{~mm}$ and a depth $>20 \mathrm{~mm}$ has been reported to have higher complication rates. ${ }^{5}$ New imaging modalities are increasingly being used to manage AAS. For example, electrocardiogram gating CT imaging helps in differentiating true intimal flaps from pulsation artefacts. Prospective gating has the advantage of reduced radiation 
exposure as it is performed only during diastole, as the proximal aorta has the least amount of motion during this phase. This contrasts with retrospective gating in which images are captured throughout the cardiac cycle.

In conclusion, newer imaging modalities have increased awareness about unusual and complex AAS entities. ${ }^{4}$ Increasing one's understanding of these should enhance confidence when managing such complex cases.

Conflicts of interest None declared.

Editorial responsibility This submission was handled by Dr. Hilary P. Grocott, Editor-in-Chief, Canadian Journal of Anesthesia.

\section{References}

1. Makhija N, Magoon $R$, Sarkar S. Transesophageal echocardiographic imaging of an aortic intramural hematoma: characterizing the crescent. Can J Anesth 2019; DOI: https://doi. org/10.1007/s12630-019-01456-y.

2. Mishra AK, Nadadur S, Sahu KK, Lal A. Penetrating aortic ulcer masquerading as acute coronary syndrome. Am J Med Sci 2019; DOI: https://doi.org/10.1016/j.amjms.2019.04.027.

3. Gutschow SE, Walker CM, Martinez-Jimenez S, Rosado-deChristenson ML, Stowell J, Kunin JR. Emerging concepts in intramural hematoma imaging. Radiographics 2016; 36: 660-74.

4. Kitai T, Kaji S, Yamamuro A, et al. Impact of new development of ulcer-like projection on clinical outcomes in patients with type B aortic dissection with closed and thrombosed false lumen. Circulation 2010; 122(11 Suppl): S74-80.

5. Patatas $K$, Shrivastava V, Ettles DF. Penetrating atherosclerotic ulcer of the aorta: a continuing debate. Clin Radiol 2013; 68: 7539.

Publisher's Note Springer Nature remains neutral with regard to jurisdictional claims in published maps and institutional affiliations. 